\title{
Symptomatologic Analysis of Stroke in Stroke-Prone SHR
}

\author{
Ichiro Akiguchi, ${ }^{*}$ Ryohichi Horie, ${ }^{\circ * *}$ Michiya Ohtaka, ${ }^{\circ * * *}$ \\ Yukio YAMORI, ${ }^{\circ * * * *}$ and Chuichi KAWAI*
}

The establishment of stroke-prone SHR (SHRSP) has enabled us to study extensively on the pathogenesis of cerebrovascular diseases. The pathogenic similarity of stroke in these SHRSP and humans has already been stressed in previous studies (Yamori et al: Stroke 7: 46, 1976). However, there have been no adequate approaches to clarify the symptomatology of stroke in rats and so the detailed sings and symptoms of stroke in SHRSP have not been analyzed. In the present study we attempted a neurological analysis of stroke in SHRSP and then compared the results with those in humans.

Materials and Methods:

We performed neurological examinations to observe the full "clinical course" of SHRSP showing various signs and symptom of stroke. Before carrying out the survey, we made up the neurological protocol to note these signs and symptomes in SHRSP. Thus, after intensive follow-up on neurological status and lethal course, we autopsied about 50 SHRSP, which either died spontaneously or were sacrificed at the terminal stage of stroke. Then we conducted macroscopical and microscopical examinations in order to evaluate the relation between "clinical findings" and pathological findings.

Results:

1. Symptomatology of stroke in SHRSP

SHRSP with stroke lesions showed various signs and symptoms. For the purpose of description, these were roughly classified into the following 3 groups:

A) Behavioral and mental status (Yamori's classification)

Stage 1-normal,

Stage 2-irritable; hyperirritability, aggressiveness, self-biting, piloerection and ear erection to various stimuli, hyperkinesia, repetitive lifting of paws, jumping, and escaping,

Stage 3-lethargic; tendency to lethargia and hypokinesia, and hyperirritability to painful or arousal stimuli,

Stage 4-akinetic; akinesia, lethargia, and hyporesponsiveness to painful or arousal stimuli.

B) General signs

Head enlargement, self-biting, fur atrophy, incontinence, emaciation, and general weakness.

C) Focal signs

1) Motor function, motor response by Yamori's tail torsion test (YTT test); Monoparesis, hemiparesis, paraparesis, double hemiparesis,

From the Japan Stroke Prevention Center (JSPG), Izumo; Departments of Internal Medicine, * Neurosurgery,** and Pathology,**** Faculty of Medicine, Kyoto University, Kyoto; the Center for Adult Disease, *** Osaka. 
Table I. Topographical Correlation of Stroke in SHRSP

\begin{tabular}{|c|c|c|c|c|c|c|c|c|c|c|c|}
\hline \multirow{3}{*}{ Focal Signs } & \multirow{2}{*}{\multicolumn{3}{|c|}{$\begin{array}{l}\text { Behavioral } \\
\text { Status }\end{array}$}} & \multicolumn{7}{|c|}{ Pathological Findings } & \multirow{3}{*}{$\begin{array}{l}\text { Topographic- } \\
\text { al Corre- } \\
\text { spondence }\end{array}$} \\
\hline & & & & \multicolumn{3}{|c|}{ Severity } & \multicolumn{3}{|c|}{ Diagnosis } & \multirow{2}{*}{$\begin{array}{l}\text { Cerebellar } \\
\text { Herniation }\end{array}$} & \\
\hline & 2 & 3 & 4 & A & B & C & $\mathrm{H}$ & I & C & & \\
\hline $\begin{array}{l}\text { No focal signs } \\
6 / 20\end{array}$ & $6 / 6$ & 0 & 0 & $5 / 6$ & $1 / 6$ & 0 & 1 & 3 & 3 & 0 & \\
\hline $\begin{array}{l}\text { Crural paresis } \\
4 / 20\end{array}$ & $4 / 4$ & 0 & 0 & 0 & $4 / 4$ & 0 & 2 & 4 & 0 & $4 / 4$ & $\begin{array}{c}3 / 4 \\
(75 \%)\end{array}$ \\
\hline $\begin{array}{l}\text { Hemiparesis } \\
7 / 20\end{array}$ & $5 / 7$ & $2 / 7$ & 0 & $3 / 7$ & $4 / 7$ & 0 & 1 & 2 & 4 & $\begin{array}{c}4 / 6 \\
1 \text {; unknown }\end{array}$ & $\begin{array}{c}6 / 7 \\
(86 \%)\end{array}$ \\
\hline $\begin{array}{l}\text { Quadriparesis } \\
3 / 20\end{array}$ & 0 & $1 / 3$ & $2 / 3$ & 0 & $1 / 3$ & $2 / 3$ & 1 & 2 & 2 & $3 / 3$ & $\begin{array}{c}3 / 3 \\
(100 \%)\end{array}$ \\
\hline Total & $15 / 20$ & $3 / 20$ & $2 / 20$ & $8 / 20$ & $10 / 20$ & $2 / 20$ & 5 & 11 & 9 & $11 / 19$ & $12 / 14 \quad(86 \%)$ \\
\hline
\end{tabular}

A : mild, $B$ : moderate, $\mathrm{C}$ : severe, $\mathrm{H}$ : hemorrhage, $\mathrm{I}:$ infarction, $\mathrm{C}:$ cyst

and quadriparesis.

2) Involuntary movement, gait and posture.

3) Sensory disturbance; behavioral response to painful stimuli.

2. "Clinical course" of stroke in SHR

Most typically, SHRSP with stroke lesions manifested the following stages:

A) Initial stage-hyperirritability, hyperkinesia, and mild head enlargement,

B) Impending stage-hyperirritability, self-biting, moderate head enlargement, weakness, and paresis (monoparesis or hemiparesis),

C) Late or terminal stage-lethargic, akinetic, marked head enlargement, emaciation, incontinence, general weakness, paresis (para, double hemi, or quadriparesis), and abnormal respiratory patterns.

3. Topographical correlation of stroke in SHRSP

Among autopsied cases, 20 sacrificed SHRSP were examined more intensively for close inspection of the brain and spinal cord by cutting gross slices and then making histological sections in order to clarify the topographical correlation.

Results are summarized as follows (see Table I):

1) Laterality in neurological manifestation well corresponded either grossly or microscopically to the contralateral site of the lesions in the brain.

2) The severity of behavioral status or focal signs also correlated well with the severity of lesions and cerebellar herniation.

Summary with Discussion:

Close observation of signs and symptoms in SHRSP enabled us to analyze to some extent the pathology and topography of cerebrovascular lesions. The present study raised the importance of symptomatology in SHRSP, and also reassured that SHRSP is a good animal model for stroke from a symptomatological view point.

This study was supported by the Science and Technology Agency of Japanese Government, Ministry of Education, National Institutes of Health, USA (Grant HL 17754), Japan Tabacco and Salt Public Corporation, and Japan Heart Foundation. 